\title{
Open Reduction and Internal Fixation of Isolated Posterior Cruciate Ligament Avulsion Fractures: Clinical and Functional Outcome
}

\author{
Sachin Joshi, $\mathrm{MD}^{1}$, Chirag Bhatia, $\mathrm{MD}^{1,2}$, Ashwin Gondane, $\mathrm{MD}^{1}$, Avinash Rai, $\mathrm{MD}^{1}$, Sumer Singh, $\mathrm{MD}^{1,3}$, and \\ Shobhit Gupta, MD ${ }^{1}$ \\ ${ }^{1}$ Department of Orthopaedics, Government Medical College, Kota, Rajasthan; ${ }^{2}$ Department of Orthopaedics, Cygnus Orthocare Hospital, New Delhi; ${ }^{3}$ Department of \\ Orthopaedics, American International Institute of Medical Sciences, Udaiour, Rajasthan, India
}

\begin{abstract}
Purpose: The posterior cruciate ligament (PCL) presents commonly with avulsion fractures of its tibial attachment. An avulsion fracture of the PCL, if not surgically fixed, may lead to secondary changes in the knee joint. Various fixation techniques have been explored with use of lag screws, steel wires, absorbable screws, suture anchors and straddle nails. The purpose of this study was to evaluate the clinical and functional outcome of open reduction and internal fixation of tibial avulsion injuries of the PCL using cannulated cancellous screws.

Materials and Methods: We performed open reduction and internal fixation using cannulated cancellous screws in 14 patients (mean age, 33.9 years) with isolated PCL avulsion injuries. Patients with a duration of injury more than 12 weeks were excluded. The minimum follow-up period was 12 months. Results were assessed radiologically and clinically. Final functional outcome was assessed using the Lysholm knee scoring system.

Results: The average follow-up period was 13.5 months. At the final follow-up, all 14 patients achieved fracture union. The average flexion was $121.7^{\circ} \pm 9.2^{\circ}$ with full extension achieved in all patients. Mild instability $(1+)$ was noted in 4 patients. The Lysholm functional score was excellent in 11 patients, good in 2 patients and fair in 1 patient with an average score of $97 \pm 7.6$.

Conclusions: Stable early fixation for PCL avulsion injuries with early controlled mobilization provided excellent to good results. However, fixation should not be discouraged for late-presenting patients because good to fair results can be achieved in such cases.
\end{abstract}

Keywords: Knee, Posterior cruciate ligament, Fraucture, Avulsion, Fixation

\section{Introduction}

Isolated posterior cruciate ligament (PCL) injuries are uncommon and often go undiagnosed in acutely injured knees ${ }^{1)}$. The PCL, being a strong ligament, presents more commonly with avulsion fractures of its tibial attachment ${ }^{2)}$ unlike the anterior cruciate ligament whose tears commonly present as isolated le-

Received March 12, 2017; Revised June 8, 2017; Accepted June 12, 2017 Correspondence to: Chirag Bhatia, MD

Department of Orthopaedics, Cygnus Orthocare Hospital, C-5/29, Safdarjung Development Area, Hauz Khas, Opp. IIT Main Gate, New Delhi 110016, India

Tel: +91-99-6884-9269, Fax: +91-99-6884-9269

E-mail: drbhatiachirag@gmail.com

This is an Open Access article distributed under the terms of the Creative Commons Attribution Non-Commercial License (http://creativecommons.org/licenses/by-nc/4.0/) which permits unrestricted non-commercial use, distribution, and reproduction in any medium, provided the original work is properly cited. sions. Avulsion injuries differ from other PCL injuries in that they are easily diagnosed on standard radiographs where a bony fragment may be visible. Traditionally, surgery of PCL injuries was deferred due to an apprehension that the approach to the posterior part of the knee is difficult ${ }^{3)}$. However, surgical fixation of avulsion injuries is recommended to avoid morbidity associated with nonunion of the fracture ${ }^{4)}$.

Repair of avulsion injuries can be done with an open or arthroscopic technique. Biomechanical studies have also demonstrated comparable results of screw fixation using open or arthroscopic means ${ }^{5)}$. However, arthroscopic repair is technically more challenging, requires specialized equipment and has a long learning curve. Thus, it unsuitable to perform in primary centers. Open reduction and internal fixation using screws has been considered a favorable method to manage PCL avulsion injuries producing satisfactory results ${ }^{6-10)}$. In addition, a simplified posteromedial approach described by Burks and Schaffer ${ }^{11)}$, which is 
useful for avoiding difficulties associated with previous posterior approaches to the knee, has become the standard approach to the PCL.

There is currently a wide variety of materials available for internal fixation, including lag screws, steel wires, absorbable screws, suture anchors and straddle nails ${ }^{12,13)}$. Fixation with screws has shown favorable results; however, no one technique has been considered a gold standard for avulsion fractures of the PCL. In this study, we present our experience with fixation using cannulated cancellous screws, which is a good implant to achieve compression needed to fix avulsion fractures and is easily available. The approach we used was also simple and helpful in avoiding the neurovascular bundles unlike other posterior approaches.

The purpose of our study was to evaluate the clinical and functional outcome after open reduction and internal fixation of tibial avulsion injuries of the PCL using cannulated cancellous screws. We hypothesized that the technique would provide improved clinical and functional outcome with use of an easily available implant and a simple approach.

\section{Materials and Methods}

Twenty-one patients with PCL avulsion fractures were enrolled for the study from December, 2012 to November, 2015. Patients aged between 18 and 60 years with a duration of injury less than 12 weeks were included in the study. Patients with any other associated ligament injury of the same joint, any other bony injury of the same limb or any medical comorbidity precluding the sur-

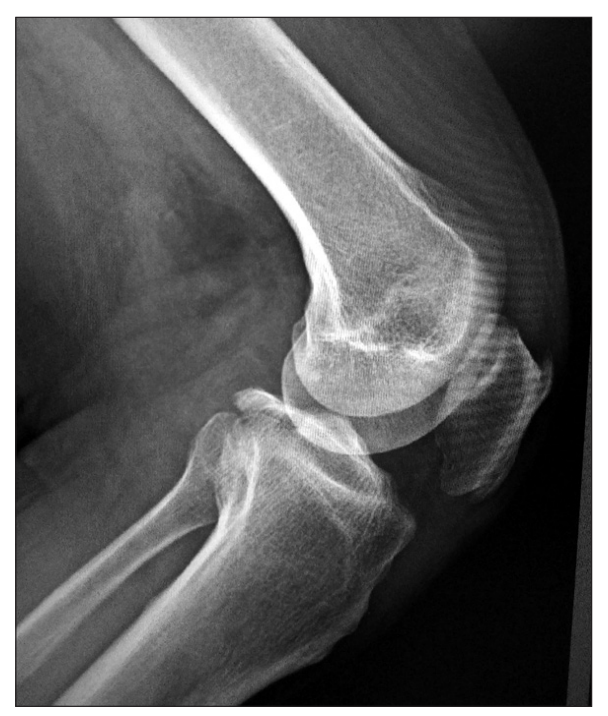

Fig. 1. Lateral radiograph of the knee (non-stress view) showing the avulsed fragment from the tibia (case no. 3). gery were excluded from the study. Patients in whom the avulsed fragment was too small $\left(<20 \mathrm{~mm}^{2}\right)$ to be fixed using a screw were also excluded. After excluding 7 patients based on the above criteria, 14 patients having isolated PCL avulsion fracture were ultimately included in the study.

All patients underwent thorough clinical examination. All the cases were clinically assessed by the senior author using the drawer test to confirm PCL injury. Anteroposterior and lateral radiographs including the posterior stress view (Fig. 1) of the knee were taken to confirm the injury. A computed tomography scan

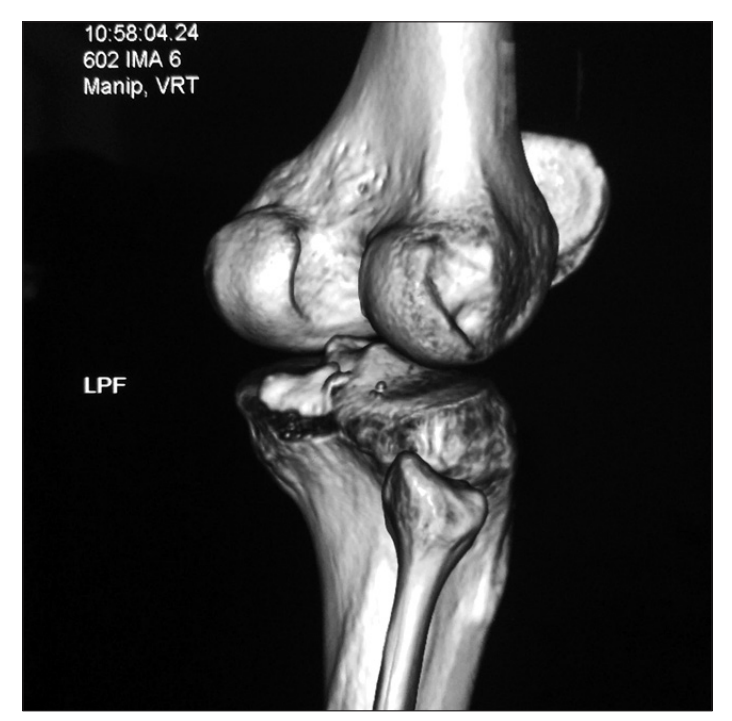

Fig. 2. Three-dimensional computed tomography scan showing the avulsed fragment of the tibia (case no. 2).

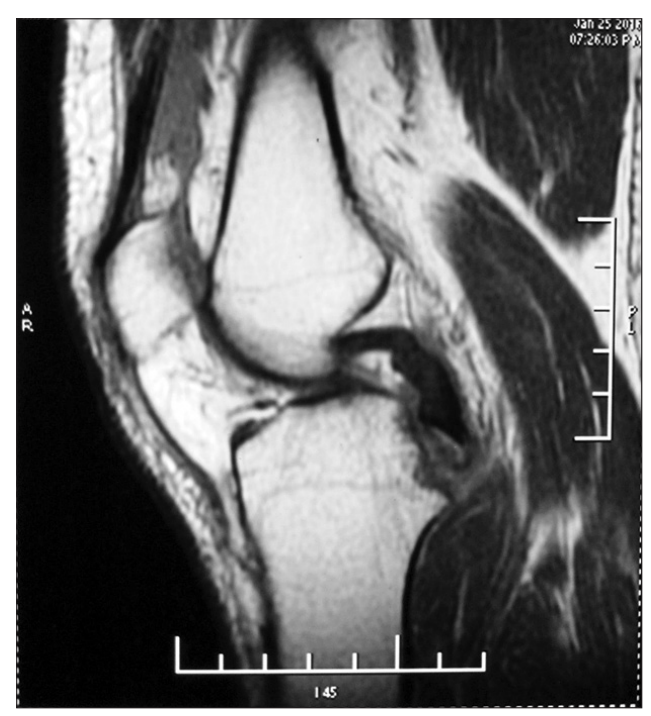

Fig. 3. Magnetic resonance imaging scan showing a lax posterior cruciate ligament avulsed from its tibial attachment (case no. 2). 


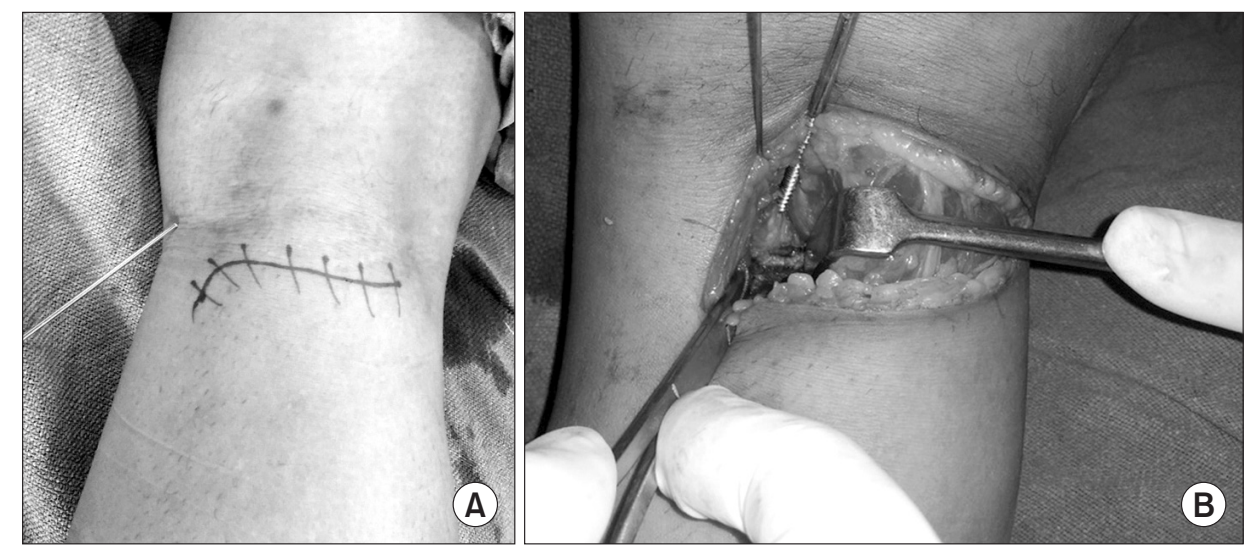

Fig. 4. Intraoperative photograph showing the skin incision and fracture site with the screw insertion technique.

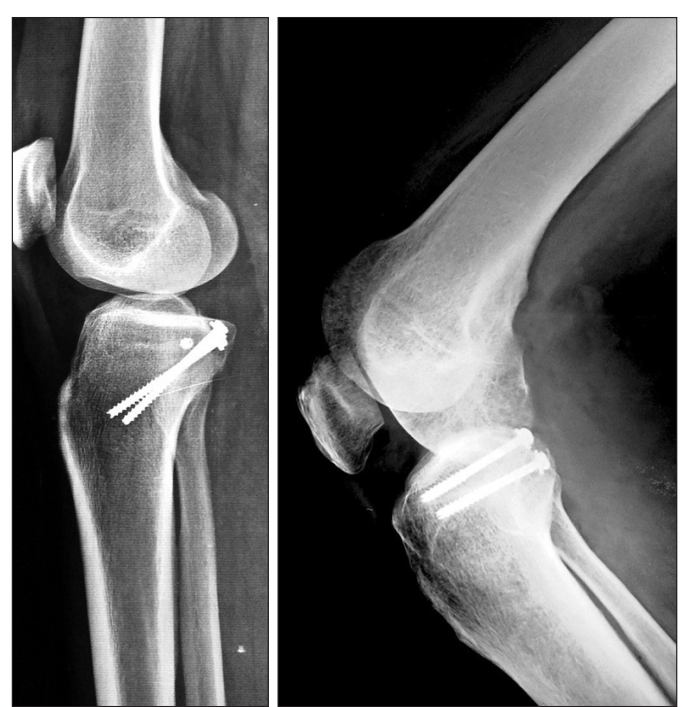

Fig. 5. Lateral radiographs of the knee showing cannulated cancellous screws in situ after fixation of the tibial avulsion of the posterior cruciate ligament in case no. 4 (A) and case no.10 (B). Note the different orientation of screws, which was decided according to the orientation of the fracture line.

(Fig. 2) was done for patients in whom the injury could not be defined well on radiographs. Magnetic resonance imaging (MRI) (Fig. 3) was done for every patient in order to confirm associated ligament injuries of the knee.

After informed consent was obtained, patients were operated using the modified posterior approach described by Burks and Schaffer ${ }^{11}$. The approach (Fig. 4) uses an inverted 'L' incision starting on the medial border of the gastrocnemius and curving along the flexor crease of the joint towards the lateral side. The fascia is incised in the line of skin incision. The capsule is exposed using the interval between the semimembranosus and gastrocnemius, which is then incised longitudinally to expose the joint at the site of the avulsed fragment. The avulsed fragment was debrided if needed and reduced using a clamp. It is of note that the reduction was achieved by slight flexion of the joint. The avulsed fragment was fixed using one or two $4 \mathrm{~mm}$ cannulated cancellous screws according to the size of the fragment (Fig. 5). A washer was used when compression was not achieved with the screw alone.

Postoperatively, the limb was kept immobilized using a long knee brace for 6 weeks. Quadriceps strengthening was started from the 2nd postoperative day. Passive knee bending was started after suture removal (10-12 days after surgery) and active knee mobilization was started 3 weeks after surgery. Partial weight bearing was allowed after 6 weeks and full weight bearing after 12 weeks when the brace was also discarded. Return to heavy activities, such as running and sports, were allowed only after 6-9 months.

Patients were regularly followed up every week for a month, then every month for the first 3 months and every 3 months thereafter for a minimum of 12 months. At every follow-up, patients were assessed clinically and radiographically. Outcomes were assessed in terms of stability and range of motion (ROM). Stability was assessed clinically by the drawer test and radiologically by lateral stress X-ray (Fig. 6). Final functional outcome was assessed using the Lysholm knee scoring system.

Statistical analysis was done using the Wilcoxon nonparametric paired test to determine the significance of postoperative improvement with respect to the Lysholm score as well as joint stability.

\section{Results}

Of the 14 patients, 12 were male and 2 were female. The mean age of the patients was 33.9 years, ranging from 22 to 54 years (Table 1). The most common mode of injury was road traffic accident $(n=9)$ with a majority involving motorcycle accident fol- 

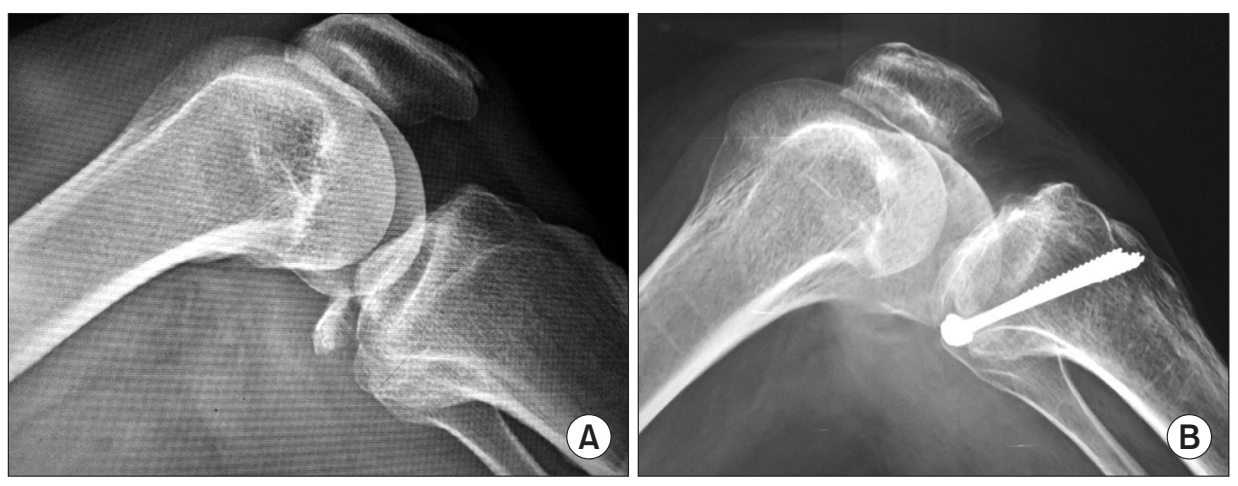

Fig. 6. Preoperative (A) and postoperative (B) posterior tibial sagging (case no. 3 ).

Table 1. Details of the 14 Cases

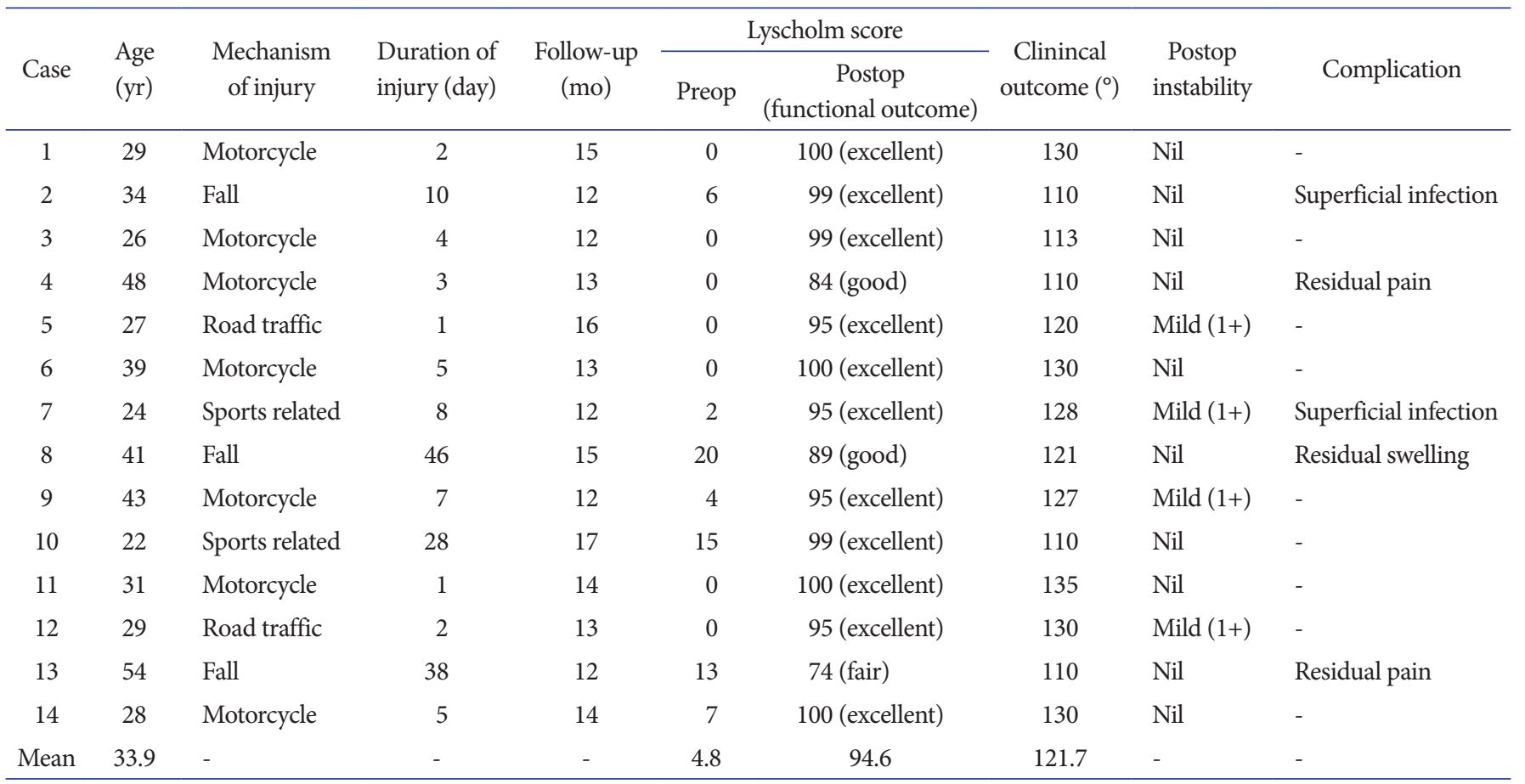

Preop: preoperative, Postop: postoperative.

lowed by either sports-related injury or fall. Eleven patients presented to the clinic within 3 weeks of injury while three patients presented between 3 to 12 weeks after injury. The average period of follow-up was 13.5 months.

At the final follow-up, all 14 patients had achieved fracture union. At 6 weeks after surgery, the ROM was more than $90^{\circ}$ in 9 patients. Of the remaining 5 patients, 3 patients who had presented later than 3 weeks after injury regained more than $90^{\circ}$ of flexion after more than 6 postoperative weeks. At the final followup, the average flexion was $121.7^{\circ} \pm 9.18^{\circ}$ with full extension possible in all the patients. The functional outcome assessed by the Lysholm scoring system was excellent in 11 patients, good in 2 patients and fair in one patient. The average Lysholm score was

\section{$97 \pm 7.6$.}

Two patients complained of residual pain in the joint while one complained of residual swelling in the joint. Two patients had superficial wound infection, which was treated with intravenous antibiotics and regular dressings. No other complications were observed.

Instability tested clinically using the drawer test and radiologically by stress X-ray was found to be mild (1+) in 4 of the patients while the rest of the patients had no residual instability.

The statistical analysis showed highly significant improvement $(p<0.001)$ in functional outcome assessed by the Lysholm score and joint stability $(\mathrm{p}<0.001)$ (Table 2$)$. 
Table 2. Statistical Analysis

\begin{tabular}{ccccc}
\hline Parameter & Preoperative & Postoperative & p-value & Inference \\
\hline Lysholm score & $3 \pm 6.7$ & $97 \pm 7.6$ & $<0.001$ & Significant \\
Instability & $2.5 \pm 0.5$ & $0.3 \pm 0.5$ & $<0.001$ & Significant \\
\hline
\end{tabular}

Values are presented as mean \pm standard deviation.

\section{Discussion}

PCL injuries account for approximately $20 \%$ of total ligament injuries of the $\mathrm{knee}^{14)}$. The incidence is especially high in cases of high-energy trauma (motorcycle and car accidents), and athletic population involved in contact sports is especially vulnerable to PCL injuries ${ }^{4,15)}$. The most common mechanism underlying PCL avulsion fractures of the tibia in road traffic accidents is dashboard collision in which a direct force is applied to the proximal part of the tibia in an anterior-to-posterior direction, with the knee in flexion ${ }^{16,17)}$. In our series, most of the injuries resulted from motorcycle accidents because the majority of people commute by two-wheelers in India.

Though the necessity of surgical treatment of isolated PCL injuries is still controversial, fixation methods for avulsion fractures of the PCL at the tibial insertion have been suggested in different series ${ }^{18-23)}$. If left untreated, the injury leads to secondary joint changes resulting in osteoarthritis ${ }^{24)}$. Open fixation through the posteromedial approach seems to be a relatively easy procedure that can be performed at any center by any orthopedic surgeon. The posteromedial approach used in our patients was devised by Burks and Schaffer ${ }^{11)}$, which is a simple approach exposing the desired surgical area without having to disturb the neurovascular bundles as with other approaches described by Abbott, Trickey, Ogata, and McCormick ${ }^{3,20,25,26)}$.

In this study, we performed open reduction and internal fixation of isolated PCL avulsion injuries using cannulated cancellous screws, assuming to attain good functional and clinical outcome. The most important findings of our study include that excellent to good functional outcome was obtained in the majority of patients (92.9\%) and fairly good results were achieved in terms of ROM as well as stability.

In our series, we treated 14 patients with isolated PCL avulsion injuries by internal fixation using cannulated cancellous screws and the results were excellent in $78.6 \%$, good in $14.3 \%$ and fair in $7.1 \%$ of the patients with an average postoperative Lysholm score of $97 \pm 7.6$. These results were superior to those reported in previous studies. In a study by Attia and Zanfaly ${ }^{27)}$ among 12 patients treated using navicular screws, excellent results were obtained in $33.3 \%$, good results in $58.3 \%$ and fair results in $8.3 \%$. Similarly,
Piedade and Mischan ${ }^{28)}$ showed excellent results in 53\% and good in $47 \%$ of their cases where screws or Polyester no. 5 were used to fix the fracture. Khatri et al. ${ }^{29)}$ used the same approach and technique, which resulted in a postoperative Lysholm score of $90.85 \pm 5.58$. In the present study, all patients attained fair to good ROM with an average flexion of $121.7^{\circ} \pm 9.2^{\circ}$ and full extension. Khatri et al. ${ }^{29)}$ achieved normal to nearly normal ROM in $96 \%$ of the patients where as $64.3 \%$ of the patients achieved results similar to our study. Postoperative knee flexion was inferior in patients who had presented to the clinic relatively late and who did not adhere to the prescribed physiotherapy. Hence early fixation and strict adherence to physiotherapy are recommended.

Four of the patients who had mild (1+) residual instability had an intrasubstance PCL injury determined on MRI. However, this instability did not affect the functional outcome. Bali et al. ${ }^{10)}$ reported that intrasubstance occult injuries were present in 15 out of 42 patients included in their study and 6 of them required a PCL reconstruction at a later stage. By contrast, none of the patients in our study had gross instability that necessitated PCL reconstruction at a later stage.

Although the technique we used in this study resulted in significant improvement in functional and clinical outcome, it is difficult to conclude that it should be the go to technique for PCL avulsion fractures due to the small sample size. Further studies with a larger sample size or case-control studies comparing different techniques are necessary to confirm our results. Nevertheless, the significance of this study is that we explored a novel and simple technique and added to the available literature on the management of PCL avulsion fractures.

\section{Conclusions}

Avulsion injuries of the PCL, though rare, should not be ignored. These injuries can be easily approached using a modified posterior approach and cancellous screw fixation with early controlled knee mobilization provides excellent to good functional results. Early diagnosis and fixation is preferred; however, fixation should not be discouraged in patients presenting late since comparably good to fair results can be obtained in such cases as well. 


\section{Conflict of Interest}

No potential conflict of interest relevant to this article was reported.

\section{References}

1. Allen CR, Kaplan LD, Fluhme DJ, Harner CD. Posterior cruciate ligament injuries. Curr Opin Rheumatol. 2002;14:142-9.

2. Sonin AH, Fitzgerald SW, Hoff FL, Friedman H, Bresler ME. MR imaging of the posterior cruciate ligament: normal, abnormal, and associated injury patterns. Radiographics. 1995;15:551-61.

3. Abbott LC, Carpenter WF. Surgical approaches to the knee joint. J Bone Joint Surgery. 1945;27:277-310.

4. Griffith JF, Antonio GE, Tong CW, Ming CK. Cruciate ligament avulsion fractures. Arthroscopy. 2004;20:803-12.

5. Sasaki SU, da Mota e Albuquerque RF, Amatuzzi MM, Pereira CA. Open screw fixation versus arthroscopic suture fixation of tibial posterior cruciate ligament avulsion injuries: a mechanical comparison. Arthroscopy. 2007;23:122630.

6. Chen $\mathrm{CH}$, Chen WJ, Shih $\mathrm{CH}$. Fixation of small tibial avulsion fracture of the posterior cruciate ligament using the double bundles pull-through suture method. J Trauma. 1999;46:1036-8.

7. Chiu FY, Wu JJ, Hsu HC, Lin L, Lo WH. Management of avulsion injury of the PCL with reattachment. Injury. 1994;25:2935.

8. Jazayeri SM, Esmaili Jah AA, Karami M. A safe posteromedial approach to posterior cruciate ligament avulsion fracture. Knee Surg Sports Traumatol Arthrosc. 2009;17:244-7.

9. Nicandri GT, Klineberg EO, Wahl CJ, Mills WJ. Treatment of posterior cruciate ligament tibial avulsion fractures through a modified open posterior approach: operative technique and 12- to 48-month outcomes. J Orthop Trauma. 2008;22:317-24.

10. Bali K, Prabhakar S, Saini U, Dhillon MS. Open reduction and internal fixation of isolated PCL fossa avulsion fractures. Knee Surg Sports Traumatol Arthrosc. 2012;20:315-21.

11. Burks RT, Schaffer JJ. A simplified approach to the tibial attachment of the posterior cruciate ligament. Clin Orthop Relat Res. 1990;(254):216-9.

12. Sun HQ, Ren YJ, Zhang YQ. Treatment of posterior cruciate ligament avulsion fracture with pressurized staples. Chi J Orthop Trauma. 2011;13:1189-90.
13. Fu YP, Hang CM, Fam HQ. Treatment of posterior cruciate ligament avulsion fracture using anchor system combined with cannulated screw. J Pract Orthop. 2011;17:73-4.

14. Deehan DJ, Pinczewski LA. Arthroscopic reattachment of an avulsion fracture of the tibial insertion of the posterior cruciate ligament. Arthroscopy. 2001;17:422-5.

15. Wind WM Jr, Bergfeld JA, Parker RD. Evaluation and treatment of posterior cruciate ligament injuries: revisited. Am J Sports Med. 2004;32:1765-75.

16. Janousek AT, Jones DG, Clatworthy M, Higgins LD, Fu FH. Posterior cruciate ligament injuries of the knee joint. Sports Med. 1999;28:429-41.

17. Schulz MS, Russe K, Weiler A, Eichhorn HJ, Strobel MJ. Epidemiology of posterior cruciate ligament injuries. Arch Orthop Trauma Surg. 2003;123:186-91.

18. Torisu T. Isolated avulsion fracture of the tibial attachment of the posterior cruciate ligament. J Bone Joint Surg Am. 1977;59:68-72.

19. Seitz H, Schlenz I, Pajenda G, Vecsei V. Tibial avulsion fracture of the posterior cruciate ligament: K-wire or screw fixation? A retrospective study of 26 patients. Arch Orthop Trauma Surg. 1997;116:275-8.

20. Trickey EL. Rupture of the posterior cruciate ligament of the knee. J Bone Joint Surg Br. 1968;50:334-41.

21. Lee HG. Avulsion fracture of the tibial attachments of the cruciate ligaments: treatment by operative reduction. J Bone Joint Surg. 1937;19:460-8.

22. Kim SJ, Shin SJ, Choi NH, Cho SK. Arthroscopically assisted treatment of avulsion fractures of the posterior cruciate ligament from the tibia. J Bone Joint Surg Am. 2001;83:698-708.

23. Kim SJ, Shin SJ, Cho SK, Kim HK. Arthroscopic suture fixation for bony avulsion of the posterior cruciate ligament. Arthroscopy. 2001;17:776-80.

24. Strobel MJ, Weiler A, Schulz MS, Russe K, Eichhorn HJ. Arthroscopic evaluation of articular cartilage lesions in posterior-cruciate-ligament-deficient knees. Arthroscopy. 2003;19:262-8

25. Ogata K. Posterior cruciate reconstruction using iliotibial band: preliminary report of a new procedure. Arch Orthop Trauma Surg. 1983;101:107-10.

26. McCormick WC, Bagg RJ, Kennedy CW Jr, Leukens CA. Reconstruction of the posterior cruciate ligament: preliminary report of a new procedure. Clin Orthop Relat Res. 1976;(118):30-1.

27. Attia ME, Zanfaly AI. Fixation of tibial bony avulsion of the posterior cruciate ligament using the posteromedial ap- 
216 Joshi et al. Open Reduction and Internal Fixation of PCL Avulsion Fractures

proach. Egypt Orthop J 2014;49:81-5.

28. Piedade SR, Mischan MM. Surgical treatment of avulsion fractures of the knee PCL tibial insertion: experience with 21 cases. Acta Ortop Bras. 2007;15:272-5.
29. Khatri K, Sharma V, Lakhotia D, Bhalla R, Farooque K. Posterior cruciate ligament tibial avulsion treated with open reduction and internal fixation through the burks and schaffer approach. Malays Orthop J. 2015;9:2-8. 